\title{
On Ulam's type stability for a class of impulsive fractional differential equations with nonlinear integral boundary conditions
}

\author{
Arshad Alia , Faranak Rabiei ${ }^{\mathrm{b}, \mathrm{c}, *}$, Kamal Shah ${ }^{\mathrm{a}}$ \\ a Department of Mathematics, University of Malakand, Chakadara Dir(L), Khyber Pakhtunkhwa, Pakistan. \\ ${ }^{b}$ Department of Mathematics, Faculty of Science, Universiti Putra Malaysia, 43400 UPM Serdang, Selangor, Malaysia. \\ ${ }^{c}$ Institute For Mathematical Research, Universiti Putra Malaysia, 43400 UPM Serdang, Selangor, Malaysia. \\ Communicated by K. Cieplinski
}

\begin{abstract}
In this manuscript, using Schaefer's fixed point theorem, we derive some sufficient conditions for the existence of solutions to a class of fractional differential equations (FDEs). The proposed class is devoted to the impulsive FDEs with nonlinear integral boundary condition. Further, using the techniques of nonlinear functional analysis, we establish appropriate conditions and results to discuss various kinds of Ulam-Hyers stability. Finally to illustrate the established results, we provide an example. (C)2017 All rights reserved.
\end{abstract}

Keywords: Caputo fractional derivative, integral boundary conditions, impulsive condition, fixed point theorem, Ulam stability.

2010 MSC: 34A08, 35R11, 26A33.

\section{Introduction and preliminaries}

Recently impulsive differential equations have been considered by many authors due to their significant applications in various fields of science and technology. These equations describe the evolution processes that are subjected to abrupt changes and discontinuous jumps in their states. Many physical systems like the function of pendulum clock, the impact of mechanical systems, preservation of species by means of periodic stocking or harvesting and the heart's function, etc. naturally experience the impulsive phenomena. Similarly in many other situations, the evolutional processes have the impulsive behavior. For example, the interruptions in cellular neural networks, the damper's operation with percussive effects, electromechanical systems subject to relaxational oscillations, dynamical systems having automatic regulations, etc., have the impulsive phenomena. For detail study, see [1, 6, 9, 13, 29, 39]. Due to its large number of applications, this area has been received great importance and remarkable attention from the researchers. In [34], Wang et al. studied the existence and uniqueness of solutions to a class of nonlocal

\footnotetext{
${ }^{*}$ Corresponding author

Email addresses: arshad.swatpk@gmail.com (Arshad Ali), faranak_rabiei@upm.edu.my (Faranak Rabiei), kamalshah408@gmail.com (Kamal Shah)
}

doi:10.22436/jnsa.010.09.19 
Cauchy problems of the form

$$
\left\{\begin{array}{l}
C_{\mathcal{D}^{p}} u(t)=g(t, u(t)) ; \quad t \in \mathcal{J}=[0, T], t \neq t_{m}, \text { for } m=1,2, \ldots, q, \\
u(0)=u_{0},\left.\Delta(u(t))\right|_{t=t_{m}}=\left.I_{m}(u(t))\right|_{t=t_{m}}, m=1,2, \ldots, q
\end{array}\right.
$$

where ${ }^{C} \mathcal{D}^{p}$ is the Caputo fractional derivative of order $p \in(0,1)$, the function $g: \mathcal{J} \times \mathbb{R} \rightarrow \mathbb{R}$ is continuous and $\mathfrak{u}_{0} \in \mathbb{R}$. Wang et al. [32], studied a class of differential equations with fractional integrable impulses of the form

$$
\left\{\begin{array}{l}
u^{\prime}(t)=g(t, u(t)), t \in\left(\zeta_{m}, t_{m+1}\right], \quad m=1,2, \ldots, q, \\
u(t)=I_{t_{m}}^{p} \sigma_{m}(t, u(t)), \quad p \in(0,1), \quad m=1,2, \ldots, q, \\
u(0)=u_{0} \in \mathbb{R},
\end{array}\right.
$$

where $g:[0, T] \times \mathbb{R} \rightarrow \mathbb{R}$ and $\sigma_{\mathrm{m}}:\left[\mathrm{t}_{\mathrm{m}}, \zeta_{\mathrm{m}}\right] \times \mathbb{R} \rightarrow \mathbb{R}$ for all $\mathrm{m}=1,2, \ldots, \mathrm{q}$ are continuous and

$$
\mathrm{I}_{\mathrm{t}_{\mathfrak{m}}}^{\mathrm{p}} \sigma_{\mathrm{m}}(\mathrm{t}, \mathrm{u}(\mathrm{t}))=\frac{1}{\Gamma(\mathrm{p})} \int_{\mathrm{t}_{\mathfrak{m}}}^{\mathrm{t}}(\mathrm{t}-\zeta)^{p-1} \sigma_{\mathfrak{m}}(\zeta, \mathrm{u}(\zeta)) \mathrm{d} \zeta
$$

Besides from the aforesaid problems, recently by using fixed point theory, several remarkable problems have been investigated in FDEs with various boundary conditions, for detail see $[2,7,28]$ and the references therein. Sometimes in many applications like numerical analysis, optimization, mathematical biology, business mathematics, economics etc., we come across the situation where finding the exact solution is quite difficult task. Therefore stability analysis plays important role in this regard. Various kinds of stability like, exponential, Mittage-Leffler stability, etc. have been considered in many papers. Another form of stability called Ulam-type stability has been studied in many papers. This concept was introduced in the mid of 19th century and now it is a well-explored area of research. Further, about the stability of functional equations, ordinary differential equations and FDEs for some recent work, we recommend the readers to study $[3,3,14,30,36]$. Further, to know about the recent contribution, we refer to $[4,5,8,10-12,15,17,18,21,26,27,31,33,35,37,38]$.

Recently another class of fractional differential equations known as fuzzy fractional differential equations has given much attention. As in very recent years, some authors investigated the solvability results for nonlocal problems of fuzzy fractional differential systems under gh-differentiability in fuzzy metric spaces, which has been further extended to fuzzy wave equations, see [24] for detail. Similarly, the authors [23], considered fuzzy fractional partial differential equations under Caputo generalized Hukuhara differentiability and developed interesting results were obtained. The aforesaid equations have significant applications in the theory of linear viscoelasticity. Onward, the authors of [22] developed a new approach for the solutions of fuzzy differential systems (FDSs) and fuzzy partial differential equations. Similarly, in [25], authors investigated the solvability of Darboux problems for nonlinear fractional partial integro-differential equations with uncertainty under Caputo gh-fractional differentiability in the infinite domain $[0, \infty) \times[0, \infty)$. Further, they introduced some new concepts about Hyers-Ulam stability and Hyers-Ulam-Rassias stability for considered problems by using the equivalent integral forms.

Motivated by the aforesaid work, in this manuscript, we investigate the existence, uniqueness and Ulam-Hyers stability results for the following implicit impulsive fractional differential equations with nonlinear integral boundary conditions

$$
\left\{\begin{array}{l}
{ }^{C} \mathcal{D}^{p} u(t)=g\left(t, u(t),{ }^{C} \mathcal{D}^{p} u(t)\right), t \neq t_{m} \in \mathcal{J}=[0, T], \text { for } m=1,2, \ldots, q \\
u(0)=\int_{0}^{T} \frac{(T-\zeta)^{p-1}}{\Gamma(p)} \sigma(\zeta, u(\zeta)) d \zeta \\
\Delta u\left(t_{m}\right)=I_{m}\left(u\left(t_{m}\right)\right), m=1,2, \ldots, q
\end{array}\right.
$$

where ${ }^{C} \mathcal{D}^{p}$ is the Caputo fractional derivative and $p \in(0,1]$, moreover, the nonlinear functions $g, \sigma$ : $\mathcal{J} \times \mathbb{R} \rightarrow \mathbb{R}$ are continuous. Further, $\mathrm{I}_{\mathfrak{m}}: \mathbb{R} \rightarrow \mathbb{R}$ represents impulsive nonlinear mapping and $\Delta \mathfrak{u}\left(\mathrm{t}_{\mathrm{m}}\right)=$ 
$u\left(t_{m}^{+}\right)-u\left(t_{m}^{-}\right)$where $u\left(t_{m}^{+}\right)$and $u\left(t_{m}^{-}\right)$represent the right and the lift limits, respectively, at $t=t_{m}$ for $\mathrm{m}=1,2, \ldots, \mathrm{q}$.

This manuscript is organized as follows. In Section 2, we introduce some notations, definitions and auxiliary results. In Section 3, we give the uniqueness and existence results for the concerned problem (1.1). The uniqueness result is obtained via the Banach contraction principle, while the existence result is obtained by using the Schaefer's fixed point theorem. In Section 4, we investigate the Ulam-Hyers stability and Ulam-Hyers-Rassias stability results for the problem (1.1). And in Section 5, we present an example to explain the applicability of our obtained results.

\section{Background materials and preliminaries}

In this section, we recall some preliminaries materials required in this paper from [19, 20, 34, 40].

Definition 2.1. The fractional integral of order $p \in \mathbb{R}_{+}$for a function $\varphi \in \mathrm{L}^{1}\left([0, T], \mathbb{R}_{+}\right)$is defined by

$$
\mathrm{I}^{\mathrm{p}} \vartheta(\mathrm{t})=\int_{0}^{\mathrm{t}} \frac{(\mathrm{t}-\zeta)^{\mathrm{p}-1}}{\Gamma(\mathrm{p})} \vartheta(\zeta) \mathrm{d} \zeta,
$$

provided that the integral converges.

Definition 2.2. The Caputo derivative of fractional order $p$ for a function $\varphi:(0, \infty) \rightarrow R$ is defined by

$$
\mathrm{c}_{\mathcal{D}^{\mathrm{p}} \vartheta(\mathrm{t})}=\frac{1}{\Gamma(\mathrm{p}-1)} \int_{0}^{\mathrm{t}}(\mathrm{t}-\zeta)^{\mathrm{n}-\mathrm{p}-1} \vartheta^{(\mathrm{n})}(\zeta) \mathrm{d} \zeta,
$$

where $n=[p]+1$ and $[p]$ is the integer part of the real number $p$.

Lemma 2.3. For $p>0$, the following result holds

$$
\mathrm{I}^{\mathrm{pC}} \mathcal{D}^{\mathrm{p}} \vartheta(\mathrm{t})=\vartheta(\mathrm{t})-\sum_{i=0}^{n-1} \frac{\vartheta^{(i)}(0)}{i !} t^{i}, \text { where } n=[\mathrm{p}]+1 .
$$

Lemma 2.4. For $\mathrm{p}>0$, the differential equation $\mathrm{C}^{\mathrm{D}} \vartheta(\mathrm{t})=0$ has a solution in the form

$$
\vartheta(t)=e_{0}+e_{1} t+e_{2} t^{2}+e_{3} t^{3}+\cdots+e_{n-1} t^{n-1} \text {, where } e_{i} \in \mathbb{R}, i=0,1,2,3, \ldots, n-1, n=[p]+1 .
$$

Lemma 2.5. For $\mathrm{p}>0$, the following result is valid

$$
I^{p C} \mathcal{D}^{p} \vartheta(t)=e_{0}+e_{1} t+e_{2} t^{2}+e_{3} t^{3}+\cdots+e_{n-1} t^{n-1} \text {, where } e_{i} \in \mathbb{R}, i=0,1,2,3, \ldots, n-1, n=[p]+1 .
$$

Let $\mathcal{J}=[0, T], \partial_{0}=\left[0, t_{1}\right], \mathcal{J}_{1}=\left(t_{1}, t_{2}\right], J_{2}=\left(t_{2}, t_{3}\right], \ldots, \mathcal{J}_{q-1}=\left(t_{q-1}, t_{q}\right], \partial_{q}=\left(t_{q}, T\right], J^{\prime}=$ $\mathcal{J} \backslash\left\{t_{1}, t_{2}, \ldots, t_{q}\right\}$. Also for convenience use the notation $\mathcal{J}_{m}=\left(t_{m}, t_{m+1}\right]$. Further, define

$$
\mathfrak{B}=\operatorname{PC}(\mathcal{J}, \mathbb{R})=\left\{\mathfrak{u}: \mathcal{J} \rightarrow \mathbb{R}: \mathfrak{u} \in \mathrm{C}\left(\mathcal{J}_{\mathrm{m}}, \mathbb{R}\right), \mathfrak{m}=1, \ldots, \mathrm{q}\right.
$$

and there exist the left limit $\mathfrak{u}\left(t_{m}^{-}\right)$and right limit $\left.\mathfrak{u}\left(t_{m}^{+}\right), m=1,2, \ldots, q\right\}$. Obviously the space $\mathfrak{B}$ is a Banach space endowed with norm defined by $\|\mathfrak{u}\|_{P C}:=\sup _{t \in \mathcal{J}}\{|\mathfrak{u}(t)|\}$. Assume that for $w \in \mathfrak{B}, \in>0$, $\psi \geqslant 0$, and a nondecreasing function $\varphi \in \mathrm{C}\left(\mathcal{J}, \mathbb{R}_{+}\right)$, the following inequalities hold:

$$
\begin{aligned}
& \left\{\begin{array}{l}
\left|{ }^{C} \mathcal{D}^{p} w(t)-g\left(t, w(t),{ }^{C} \mathcal{D}^{p} w(t)\right)\right| \leqslant \epsilon, t \in J_{m}, m=1,2, \ldots, q, \\
\left|\triangle w\left(t_{m}\right)-I_{m}\left(w\left(t_{m}\right)\right)\right| \leqslant \epsilon, m=1,2, \ldots, q,
\end{array}\right. \\
& \left\{\begin{array}{l}
\left|{ }^{C} \mathcal{D}^{p} w(t)-g\left(t, w(t),{ }^{C} \mathcal{D}^{p}(t)\right)\right| \leqslant \varphi(t), \quad t \in \mathcal{J}_{m}, m=1,2, \ldots, q, \\
\left|\Delta w\left(t_{m}\right)-I_{m}\left(w\left(t_{m}\right)\right)\right| \leqslant \psi, m=1,2, \ldots, q,
\end{array}\right.
\end{aligned}
$$

and

$$
\left\{\begin{array}{l}
\left|{ }^{C} \mathcal{D}^{p} w(t)-g\left(t, w(t),{ }^{C} \mathcal{D}^{p} w(t)\right)\right| \leqslant \epsilon \varphi(t), t \in J_{m}, m=1,2, \ldots, q \\
\left|\triangle w\left(t_{m}\right)-I_{m}\left(w\left(t_{m}\right)\right)\right| \leqslant \epsilon \psi, m=1,2, \ldots, q .
\end{array}\right.
$$


Definition 2.6 ([33]). The problem (1.1) is Ulam-Hyers stable, if there exists a real number $c_{g, m, q, \sigma}>0$, such that for each $\epsilon>0$ and for each solution $w \in \mathfrak{B}$ of the inequality (2.1), there exists a solution $u \in \mathfrak{B}$ of the problem (1.1) such that

$$
|w(t)-u(t)| \leqslant c_{g, p, q, \sigma} \in, t \in \mathcal{J} .
$$

Definition 2.7 ([33]). The problem (1.1) is generalized Ulam-Hyers stable if there exists a real number $\theta_{g, \theta, m, q, \sigma} \in C\left(\mathbb{R}_{+}, \mathbb{R}_{+}\right), \theta_{g, \theta, m, q, \sigma}(0)=0$, such that and each $\epsilon>0$ and for each solution $w \in \mathfrak{B}$ of the inequality (2.1), there exists a solution $u \in \mathfrak{B}$ to the problem (1.1) such that

$$
|w(t)-u(t)| \leqslant \theta_{g, \theta, p, q, \sigma}(\epsilon), \quad t \in \mathcal{J} .
$$

Remark 2.8. It should be noted that Definition 2.6 implies Definition 2.7.

Definition 2.9 ([33]). The problem (1.1) is Ulam-Hyers-Rassias stable with respect to $(\varphi, \psi)$, if there exists a real number $c_{g, m, q, \sigma, \varphi}>0$, such that for each $\epsilon>0$ and for each solution $w \in \mathfrak{B}$ of the inequality (2.3), there exists a solution $u \in \mathfrak{B}$ of the problem (1.1) such that

$$
|w(t)-u(t)| \leqslant c_{g, p, q, \sigma, \varphi} \in(\psi+\varphi(t)), \quad t \in \mathcal{J} .
$$

Definition 2.10 ([33]). The problem (1.1) is generalized Ulam-Hyers-Rassias stable with respect to $(\varphi, \psi)$ if there exists a real number $c_{g, p, q, \sigma, \varphi}>0$, such that for each solution $w \in \mathfrak{B}$ of the inequality (2.2), there exists a solution $u \in \mathfrak{B}$ of the problem (1.1) such that

$$
|w(t)-u(t)| \leqslant c_{g, q, \sigma, \varphi}(\psi+\varphi(t)), \quad t \in \mathcal{J} .
$$

Remark 2.11. We remark that Definition 2.9 implies Definition 2.10.

Remark 2.12. A function $w \in \mathfrak{B}$ is a solution of the inequality (2.1) if there exists a function $\varpi \in \mathfrak{B}$ and a sequence $\varpi_{m}, m=1,2 \ldots, q$ depending on $w$, such that

(i) $|\bowtie(t)| \leqslant \epsilon,\left|\varpi_{m}\right| \leqslant \epsilon, t \in \mathcal{J}_{m}, m=1,2, \ldots, q$;

(ii) ${ }^{C} \mathcal{D}^{p} \mathcal{w}(t)=g\left(t, w(t),{ }^{C} \mathcal{D}^{p} \mathcal{w}(t)\right)+\varpi(t), t \in \mathcal{J}_{m}, m=1,2, \ldots, q$;

(iii) $\Delta w\left(t=t_{m}\right)=I_{m}\left(w\left(t_{m}\right)\right)+\varpi_{m}, t \in \mathcal{J}_{m}, m=1,2, \ldots, q$.

Remark 2.13. A function $w \in \mathfrak{B}$ is a solution of the inequality (2.2) if there exists a function $\varpi \in \mathfrak{B}$ and a sequence $\varpi_{m}, m=1,2 \ldots, q$ depending on $w$, such that

(i) $|\bowtie(t)| \leqslant \varphi(t),\left|\varpi_{m}\right| \leqslant \psi, t \in \mathcal{J}_{m}, m=1,2, \ldots, q$;

(ii) ${ }^{C} \mathcal{D}^{p} \mathcal{w}(t)=g\left(t, w(t),{ }^{C} \mathcal{D}^{p} \mathcal{W}(t)\right)+\varpi(t), t \in \mathcal{J}_{m}, m=1,2, \ldots, q$;

(iii) $\Delta w\left(t_{m}\right)=I_{m}\left(w\left(t_{m}\right)\right)+\varpi_{m}, t \in \mathcal{J}_{m}, m=1,2, \ldots, q$.

Remark 2.14. A function $w \in \mathfrak{B}$ is a solution of the inequality (2.2) if there exists a function $\varpi \in \mathfrak{B}$ and a sequence $\varpi_{m}, m=1,2 \ldots, q$ (which depends on $w$ ) such that

(i) $|\bowtie(t)| \leqslant \epsilon \varphi(t),\left|\varpi_{m}\right| \leqslant \epsilon \psi, t \in \mathcal{J}_{m}, m=1,2, \ldots, q$;

(ii) ${ }^{C} \mathcal{D}^{p} \mathcal{w}(t)=g\left(t, w(t),{ }^{C} \mathcal{D}^{p} \mathcal{w}(t)\right)+\varpi(t), t \in \mathcal{J}_{m}, m=1,2, \ldots, q$;

(iii) $\Delta w\left(t_{m}\right)=I_{m}\left(w\left(t_{m}\right)\right)+\varpi_{m}, t \in \mathcal{J}_{m}, m=1,2, \ldots, q$.

Theorem 2.15 ([16, Banach's fixed point theorem $])$. Let $\mathfrak{B}$ be a Banach space. Then any contraction mapping $\digamma: \mathfrak{B} \rightarrow \mathfrak{B}$ has a unique fixed point.

Theorem 2.16 ([16, Schaefer's fixed point theorem]). Let $\mathfrak{B}$ be a Banach space and $\digamma: \mathfrak{B} \rightarrow \mathfrak{B}$ is a completely continuous operator and the set $\mathrm{D}=\{\mathfrak{u} \in \mathfrak{B}: \mathfrak{u}=\mu \digamma \mathrm{u}, 0<\mu<1\}$ is bounded. Then $\digamma$ has a fixed point in $\mathfrak{B}$.

Theorem 2.17 ([20, Arzelà-Ascoli's theorem]). Let $\mathcal{H} \in \mathrm{C}(\mathcal{J}, \mathbb{R}), \mathcal{H}$ is relatively compact (i.e., $\overline{\mathcal{H}}$ is compact) if

1. $\mathcal{H}$ is uniformly bounded that is there exists $\varepsilon>0$, such that

$$
|\mathrm{f}(v)|<\epsilon \text { for each } \mathrm{g} \in \mathcal{H} \text { and } v \in \mathcal{J} .
$$

2. $\mathcal{H}$ is equi-continuous, that is for every $\epsilon>0$, there exists $\delta>0$ such that for any

$$
w, \bar{v} \in \mathcal{J},|v-\bar{v}| \leqslant \delta \text { implies }|\mathrm{g}(v)-\mathrm{g}(\bar{v})| \leqslant \epsilon \text { for each } \mathrm{g} \in \mathcal{H} \text {. }
$$




\section{Existence of at least one solution}

In this section, we investigate the existence and uniqueness of solution to the proposed class of impulsive integral boundary value problem of implicit fractional differential equations.

Lemma 3.1. The solution $u \in \mathrm{C}^{1}(\mathcal{J}, \mathbb{R})$ of the following fractional integral boundary value problem of impulsive differential equations for $\mathrm{y} \in \mathrm{C}(\mathcal{J}, \mathbb{R})$

$$
\left\{\begin{array}{l}
C_{\mathcal{D}^{p}} u(t)=y(t), 0<p \leqslant 1, t \neq t_{m} \in \mathcal{J}, \text { for } m=1,2, \ldots, q \\
u(0)=\int_{0}^{T} \frac{(T-\zeta)^{p-1}}{\Gamma(p)} \sigma(\zeta, u(\zeta)) d \zeta \\
\triangle u\left(t_{m}\right)=I_{m}\left(u\left(t_{m}\right), \text { where } m=1,2, \ldots, q\right.
\end{array}\right.
$$

is given by

$$
u(t)=\left\{\begin{array}{c}
\frac{1}{\Gamma(p)} \int_{0}^{t}(t-\zeta)^{p-1} y(\zeta) d \zeta+\int_{0}^{T} \frac{(T-\zeta)^{p-1}}{\Gamma(p)} \sigma(\zeta, u(\zeta)) d \zeta, t \in J_{0} \\
\int_{t_{m}}^{t} \frac{(t-\zeta)^{p-1}}{\Gamma(p)} y(\zeta) d \zeta+\sum_{i=1}^{m}\left[\int_{t_{i-1}}^{t_{i}} \frac{\left(t_{i}-\zeta\right)^{p-1}}{\Gamma(p)} y(\zeta) d \zeta+I_{i}\left(u\left(t_{i}\right)\right)\right] \\
\quad+\int_{0}^{T} \frac{(T-\zeta)^{p-1}}{\Gamma(p)} \sigma(\zeta, u(\zeta)) d \zeta, t \in \mathcal{J}_{m}, m=1,2, \ldots, q .
\end{array}\right.
$$

Proof. Let $u$ be a solution of problem (3.1), then for any $t \in \mathcal{J}_{0}$, there exists a constant $e_{0} \in \mathbb{R}$ such that

$$
u(t)=I^{p} y(t)+e_{0}=\frac{1}{\Gamma(p)} \int_{0}^{t}(t-\zeta)^{p-1} y(\zeta) d \zeta+e_{0}
$$

Using the condition $u(0)=\int_{0}^{T} \frac{(T-\zeta)^{p-1}}{\Gamma(p)} \sigma(\zeta, u(\zeta)) d \zeta$, equation (3.3) yields that

$$
e_{0}=u(0)=\int_{0}^{T} \frac{(T-\zeta)^{p-1}}{\Gamma(p)} \sigma(\zeta, u(\zeta)) d \zeta
$$

Therefore, (3.3) takes the form

$$
u(t)=\frac{1}{\Gamma(p)} \int_{0}^{t}(t-\zeta)^{p-1} y(\zeta) d \zeta+\int_{0}^{T} \frac{(T-\zeta)^{p-1}}{\Gamma(p)} \sigma(\zeta, u(\zeta)) d \zeta, t \in J_{0} .
$$

Similarly for $t \in \mathcal{J}_{1}$, there exists a constant $d_{1} \in \mathbb{R}$, such that

$$
u(t)=\frac{1}{\Gamma(p)} \int_{t_{1}}^{t}(t-\zeta)^{p-1} y(\zeta) d \zeta+d_{1}
$$

Hence we have

$$
u\left(t_{1}^{-}\right)=\frac{1}{\Gamma(p)} \int_{0}^{t_{1}}\left(t_{1}-\zeta\right)^{p-1} y(\zeta) d \zeta+\int_{0}^{T} \frac{(T-\zeta)^{p-1}}{\Gamma(p)} \sigma(\zeta, u(\zeta)) d \zeta, \quad u\left(t_{1}^{+}\right)=d_{1}
$$

In view of

$$
\Delta u\left(t_{1}\right)=u\left(t_{1}^{+}\right)-u\left(t_{1}^{-}\right)=I_{1}\left(u\left(t_{1}\right)\right)
$$

we get

$$
d_{1}=\frac{1}{\Gamma(p)} \int_{0}^{t_{1}}\left(t_{1}-\zeta\right)^{p-1} y(\zeta) d \zeta+\int_{0}^{T} \frac{(T-\zeta)^{p-1}}{\Gamma(p)} \sigma(\zeta, u(\zeta)) d \zeta+I_{1}\left(u\left(t_{1}\right)\right)
$$


For this value of $d_{1}$, we have

$$
\begin{aligned}
u(t)= & \frac{1}{\Gamma(p)} \int_{t_{1}}^{t}(t-\zeta)^{p-1} y(\zeta) d \zeta+\frac{1}{\Gamma(p)} \int_{0}^{t_{1}}\left(t_{1}-\zeta\right)^{p-1} y(\zeta) d \zeta+I_{1}\left(u\left(t_{1}\right)\right) \\
& +\int_{0}^{T} \frac{(T-\zeta)^{p-1}}{\Gamma(p)} \sigma(\zeta, u(\zeta)) d \zeta, t \in J_{1} .
\end{aligned}
$$

Repeating the same fashion in this way for $t \in \mathcal{J}_{m}$, we get

$$
\begin{aligned}
u(t)= & \int_{t_{m}}^{t} \frac{(t-\zeta)^{p-1}}{\Gamma(p)} y(\zeta) d \zeta+\sum_{i=1}^{m}\left[\int_{t_{i-1}}^{t_{i}} \frac{\left(t_{i}-\zeta\right)^{p-1}}{\Gamma(p)} y(\zeta) d \zeta+I_{i}\left(u\left(t_{i}\right)\right)\right] \\
& +\int_{0}^{T} \frac{(T-\zeta)^{p-1}}{\Gamma(p)} \sigma(\zeta, u(\zeta)) d \zeta, t \in J_{m}, m=1,2, \ldots, q .
\end{aligned}
$$

Conversely, assume that $u$ is a solution of the integral equation (3.2), then we can easily verify that the solution $u(t)$ given by equation (3.2) satisfies problem (3.1) along with its impulsive and integral boundary conditions.

For obtaining the desired results, we assume the following.

$\left(\mathrm{H}_{1}\right)$ the function $\mathrm{g}: \mathcal{J} \times \mathbb{R} \times \mathbb{R} \rightarrow \mathbb{R}$ is continuous;

$\left(\mathrm{H}_{2}\right)$ there exist constants $\mathrm{K}_{\mathrm{g}}>0$ and $0<\mathrm{L}_{\mathrm{g}}<1$ such that

$$
\left|g\left(t, u_{1}, w_{1}\right)-g\left(t, u_{2}, w_{2}\right)\right| \leqslant K_{g}\left|u_{1}-u_{2}\right|+L_{g}\left|w_{1}-w_{2}\right| \text {, for } t \in \mathcal{J} \text { and } u_{1}, u_{2}, w_{1}, w_{2} \in \mathbb{R} ;
$$

$\left(\mathrm{H}_{3}\right)$ there exists a constant $\mathrm{L}_{\sigma}>0$ such that

$$
\left|\sigma\left(t, u_{1}\right)-\sigma\left(t, u_{2}\right)\right| \leqslant L_{\sigma}\left|u_{1}-u_{2}\right|, \text { for each } u_{1}, u_{2} \in \mathbb{R}, t \in \mathcal{J}_{m}, m=1,2, \ldots, q ;
$$

$\left(\mathrm{H}_{4}\right)$ there exists a constant $\mathrm{L}_{\mathrm{I}}>0$ such that

$$
\left|I_{m}\left(u_{1}\right)-I_{m}\left(u_{2}\right)\right| \leqslant L_{I}\left|u_{1}-u_{2}\right| \text { for each } u_{1}, u_{2} \in \mathbb{R}, t \in \mathcal{J}_{m} \text { and } m=1,2, \ldots, q ;
$$

$\left(\mathrm{H}_{5}\right)$ there exist constants $\alpha, \beta, \gamma \in \mathrm{C}\left(\mathcal{J}, \mathbb{R}_{+}\right)$, such that

$$
|f(t, u(t), w(t))| \leqslant \alpha(t)+\beta(t)|u|+\gamma(t)|w|, \quad \text { for } t \in \mathcal{J}, u, w \in \mathbb{R},
$$

with $\alpha^{*}=\sup _{t \in \mathcal{J}} \alpha(t), \beta^{*}=\sup _{t \in \mathcal{J}} \beta(t)$ and $\gamma^{*}=\sup _{t \in \mathcal{J}} \gamma(t)<1 ;$

$\left(\mathrm{H}_{6}\right)$ the functions $\mathrm{I}_{\mathrm{m}}: \mathbb{R} \rightarrow \mathbb{R}$ are continuous and there exist constants $\mathrm{N}, \mathrm{N}^{*}>0$, such that

$$
\left|I_{m}(u)\right| \leqslant N|u(t)|+N^{*} \text { for each } u \in \mathbb{R}, m=1,2, \ldots, q ;
$$

$\left(\mathrm{H}_{7}\right)$ the function $\sigma: \mathcal{I} \times \mathbb{R} \rightarrow \mathbb{R}$ is continuous and there exists a constant $\mathfrak{I}>0$ such that

$$
|\sigma(t, u)| \leqslant \mathfrak{I} \text { for each } t \in \mathcal{J}_{m} \text {, for all } u \in \mathbb{R} .
$$

Theorem 3.2. Let assumptions $\left(H_{1}\right)-\left(H_{4}\right)$ be satisfied and if

$$
\left[\frac{\mathrm{K}_{\mathrm{g}} \mathrm{T}^{\mathrm{p}}}{\left(1-\mathrm{L}_{\mathrm{g}}\right) \Gamma(\mathrm{p}+1)}+\frac{\mathrm{L}_{\sigma} \mathrm{T}^{\mathrm{p}}}{\Gamma(\mathrm{p}+1)}+\mathrm{q}\left(\frac{\mathrm{K}_{\mathrm{g}} \mathrm{T}^{\mathrm{p}}}{\left(1-\mathrm{L}_{\mathrm{g}}\right) \Gamma(\mathrm{p}+1)}+\mathrm{L}_{\mathrm{I}}\right)\right]<1,
$$

then the problem (1.1) has a unique solution $u$ in $\mathfrak{B}$. 
Proof. We define a mapping $\digamma: \mathfrak{B} \rightarrow \mathfrak{B}$ by

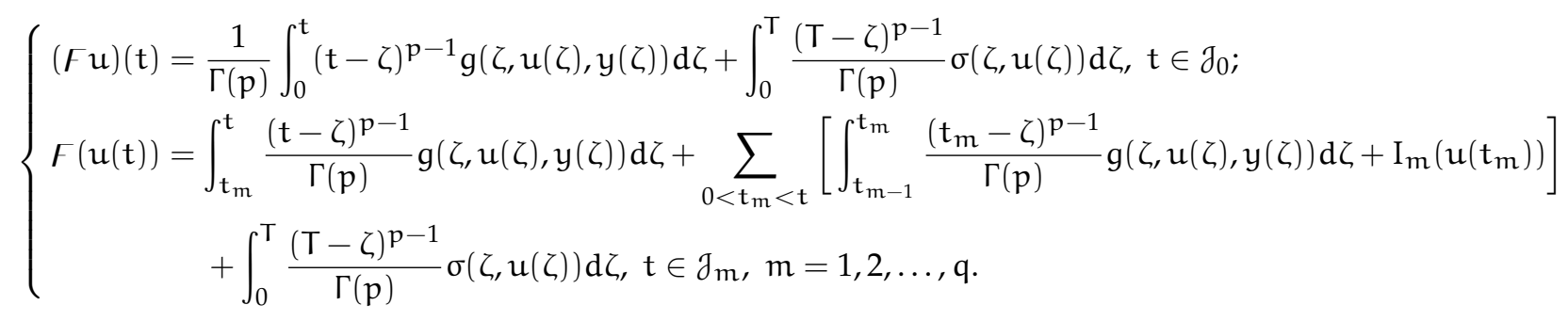

For any $u, w \in \mathfrak{B}$ and $t \in \mathcal{J}_{\mathfrak{m}}$, consider the following

$$
\begin{aligned}
& |(\digamma w)(t)-(\digamma \mathfrak{u})(\mathrm{t})| \leqslant \int_{\mathrm{t}_{\mathrm{m}}}^{\mathrm{t}} \frac{(\mathrm{t}-\zeta)^{p-1}}{\Gamma(p)}|x(\zeta)-\mathrm{y}(\zeta)| \mathrm{d} \zeta+\sum_{0<\mathrm{t}_{\mathrm{m}}<\mathrm{t}} \int_{\mathrm{t}_{\mathrm{m}-1}}^{\mathrm{t}_{\mathrm{m}}} \frac{\left(\mathrm{t}_{\mathrm{m}}-\zeta\right)^{p-1}}{\Gamma(p)}|x(\zeta)-\mathrm{y}(\zeta)| \mathrm{d} \zeta \\
& +\sum_{0<t_{\mathfrak{m}}<t}\left|I_{m}\left(w\left(t_{m}\right)\right)-I_{m}\left(u\left(t_{m}\right)\right)\right|+\int_{0}^{T} \frac{(T-\zeta)^{p-1}}{\Gamma(p)}|\sigma(\zeta, w(\zeta))-\sigma(\zeta, u(\zeta))| d \zeta,
\end{aligned}
$$

where $x, y \in C(J, \mathbb{R})$ and are given by

$$
x(t)=g(t, w(t), x(t)), \quad y(t)=g(t, u(t), y(t)) .
$$

By $\left(\mathrm{H}_{2}\right)$, we have

$$
|x(t)-y(t)|=|g(t, w(t), x(t))-g(t, u(t), y(t))| \leqslant K_{g}|w(t)-u(t)|+L_{g}|x(t)-y(t)| .
$$

Then

$$
|x(t)-y(t)| \leqslant \frac{K_{g}}{1-L_{g}}|w(t)-u(t)| .
$$

Thus using inequality (3.5) and assumptions $\left(\mathrm{H}_{3}\right)$ and $\left(\mathrm{H}_{4}\right)$, we have

$$
\begin{aligned}
\|\digamma w-\digamma u\|_{P C} \leqslant & \frac{K_{g}}{1-L_{g}} \int_{t_{m}}^{t} \frac{(t-\zeta)^{p-1}}{\Gamma(p)}|w(t)-u(t)| d \zeta+\frac{K_{g}}{1-L_{g}} \sum_{m=1}^{q} \int_{t_{m-1}}^{t_{m}} \frac{\left(t_{m}-\zeta\right)^{p-1}}{\Gamma(p)}|w(t)-u(t)| d \zeta \\
& +L_{I} \sum_{m=1}^{q}|w(t)-u(t)|+L_{\sigma} \int_{0}^{T} \frac{(T-\zeta)^{p-1}}{\Gamma(p)}|w(t)-u(t)| d \zeta \\
& \leqslant\left(\frac{K_{g} T^{p}}{\left(1-L_{g}\right) \Gamma(p+1)}+\frac{q K_{g} T^{p}}{\left(1-L_{g}\right) \Gamma(p+1)}+q L_{I}+\frac{L_{\sigma} T^{p}}{\Gamma(p+1)}\right)|w(t)-u(t)| .
\end{aligned}
$$

Thus we have

$$
\|\digamma w-\digamma u\|_{P C} \leqslant\left[\frac{K_{g} T^{p}}{\left(1-L_{g}\right) \Gamma(p+1)}+\frac{L_{\sigma} T^{p}}{\Gamma(p+1)}+q\left(\frac{K_{g} T^{p}}{\left(1-L_{g}\right) \Gamma(p+1)}+L_{I}\right)\right]\|w-u\|_{P C} .
$$

By the same process, for $t \in \mathcal{J}_{0}$, we obtain the following result

$$
\|\digamma w-\digamma u\|_{P C} \leqslant\left(\frac{K_{g} T^{p}}{\left(1-L_{g}\right) \Gamma(p+1)}+\frac{L_{\sigma} T^{p}}{\Gamma(p+1)}\right)\|w-u\|_{P C} .
$$

Combining both the results, we have for $t \in \mathcal{J}_{0} \cup \mathcal{J}_{m}=\mathcal{J}$

$$
\|\digamma w-\digamma u\|_{P C} \leqslant\left[\frac{K_{g} T^{p}}{\left(1-L_{g}\right) \Gamma(p+1)}+\frac{L_{\sigma} T^{p}}{\Gamma(p+1)}+q\left(\frac{K_{g} T^{p}}{\left(1-L_{g}\right) \Gamma(p+1)}+L_{I}\right)\right]\|w-u\|_{P C} .
$$

Now since

$$
\left[\frac{\mathrm{K}_{\mathrm{g}} \mathrm{T}^{\mathrm{p}}}{\left(1-\mathrm{L}_{\mathrm{g}}\right) \Gamma(\mathrm{p}+1)}+\frac{\mathrm{L}_{\sigma} \mathrm{T}^{\mathrm{p}}}{\Gamma(\mathrm{p}+1)}+\mathrm{q}\left(\frac{\mathrm{K}_{\mathrm{g}} \mathrm{T}^{\mathrm{p}}}{\left(1-\mathrm{L}_{\mathrm{g}}\right) \Gamma(\mathrm{p}+1)}+\mathrm{L}_{\mathrm{I}}\right)\right]<1 \text {, }
$$

hence by the Banach's contraction theorem $\digamma$ is a contraction and thus it has a unique fixed point, which is the corresponding unique solution of problem (1.1). This completes the proof. 
Our next result is based on the Schaefer's fixed point theorem.

Theorem 3.3. If the assumptions $\left(\mathrm{H}_{1}\right)-\left(\mathrm{H}_{6}\right)$ are satisfied and if

$$
q N+\frac{\beta^{*} T^{p}(q+1)}{\left(1-\gamma^{*}\right) \Gamma(p+1)}<1,
$$

then problem (3.1) has at least one solution.

Proof. Consider the operator $\digamma$ defined in Theorem 3.2. We use the Schaefer's fixed point theorem to show that $\digamma$ has a fixed point. The proof is completed in four steps.

Step 1. $\digamma$ is continuous. Take a sequence $\left\{u_{n}\right\} \in \mathfrak{B}$, such that $u_{n} \rightarrow \mathfrak{u} \in \mathfrak{B}$. For $t \in \mathcal{J}_{m}$, we have

$$
\begin{aligned}
& \left|\left(\digamma u_{n}\right)(t)-(\digamma u)(t)\right| \\
& \quad \leqslant \int_{t_{m}}^{t} \frac{(t-\zeta)^{p-1}}{\Gamma(p)}\left|y_{n}(\zeta)-y(\zeta)\right| d \zeta+\sum_{0<t_{m}<t} \int_{t_{m}-1}^{t_{m}} \frac{\left(t_{m}-\zeta\right)^{p-1}}{\Gamma(p)}\left|y_{n}(\zeta)-y(\zeta)\right| d \zeta \\
& \quad+\sum_{0<t_{m}<t}\left|I_{m}\left(u_{n}\left(t_{m}\right)\right)-I_{m}\left(u\left(t_{m}\right)\right)\right|+\int_{0}^{T} \frac{(T-\zeta)^{p-1}}{\Gamma(p)}\left|\sigma\left(\zeta, u_{n}(\zeta)\right)-\sigma(\zeta, u(\zeta))\right| d \zeta,
\end{aligned}
$$

where $y_{n}, y \in C(\mathcal{J}, \mathbb{R})$ and are given by

$$
y_{n}(t)=g\left(t, u_{n}(t), x(t)\right) \text { and } y(t)=g(t, u(t), y(t))
$$

By $\left(\mathrm{H}_{2}\right)$, we have

$$
\left|y_{n}(t)-y(t)\right|=\left|g\left(t, u_{n}(t), y_{n}(t)\right)-g(t, u(t), y(t))\right| \leqslant K_{g}\left|u_{n}(t)-u(t)\right|+L_{g}\left|y_{n}(t)-y(t)\right| .
$$

Therefore, we have

$$
\left|y_{n}(t)-y(t)\right| \leqslant \frac{K_{g}}{1-L_{g}}\left\|u_{n}-u\right\|_{P C} .
$$

Now since $u_{n} \rightarrow u$ as $n \rightarrow \infty$, which implies that $y_{n}(t) \rightarrow y(t)$ as $n \rightarrow \infty$ for each $t \in J_{m}$. As a consequence of Lebesgue dominated convergent theorem, the right hand side of inequality (3.6) tends to zero as $n \rightarrow \infty$, hence

$$
\left|\left(\digamma u_{n}\right)(t)-(\digamma \mathfrak{u})(t)\right| \rightarrow 0 \text { as } n \rightarrow \infty,
$$

which implies that

$$
\left\|\digamma u_{n}-\digamma u\right\|_{P C} \rightarrow 0 \text { as } n \rightarrow \infty .
$$

Similarly for $t \in \mathcal{J}_{0}$, we can easily show that

$$
\left\|\digamma u_{n}-\digamma u\right\|_{P C} \rightarrow 0 \text { as } \mathrm{n} \rightarrow \infty .
$$

Thus $\digamma$ is continuous on $\mathcal{J}$.

Step 2. $\digamma$ maps bounded sets into bounded sets in $\mathfrak{B}$. In fact we just need to show that for any positive constant $\mu$, there exists a constant $\eta>0$ such that for each $u \in B_{\mu}=\left\{u \in \mathfrak{B}:\|u\|_{P C} \leqslant \mu\right\}$, we have $\|\digamma(u)\|_{\mathrm{PC}} \leqslant \eta$. For $\mathrm{t} \in \mathcal{J}_{\mathrm{m}}$

$$
\begin{aligned}
(\digamma \mathrm{u})(\mathrm{t})= & \int_{\mathrm{t}_{\mathrm{m}}}^{\mathrm{t}} \frac{(\mathrm{t}-\zeta)^{p-1}}{\Gamma(\mathrm{p})} \mathrm{y}(\zeta) \mathrm{d} \zeta+\sum_{0<\mathrm{t}_{\mathrm{m}}<\mathrm{t}} \int_{\mathrm{t}_{\mathrm{m}-1}}^{\mathrm{t}_{\mathrm{m}}} \frac{\left(\mathrm{t}_{\mathrm{m}}-\zeta\right)^{p-1}}{\Gamma(p)} \mathrm{y}(\zeta) \mathrm{d} \zeta, \\
& +\sum_{0<\mathrm{t}_{\mathrm{m}}<\mathrm{t}} \mathrm{I}_{\mathrm{m}}\left(\mathrm{u}\left(\mathrm{t}_{\mathrm{m}}\right)\right)+\int_{0}^{T} \frac{(T-\zeta)^{p-1}}{\Gamma(p)} \sigma(\zeta, u(\zeta)) \mathrm{d} \zeta,
\end{aligned}
$$


where $y \in C(\mathcal{J}, \mathbb{R})$ and is given by

$$
y(t)=g(t, u(t), y(t)) .
$$

Keeping in mind $\left(\mathrm{H}_{5}\right)$ and for $t \in \mathcal{J}_{m}$, consider

$$
|y(t)|=|g(t, u, y(t))| \leqslant \alpha(t)+\beta(t)\|u\|_{P C}+\gamma(t)|y(t)| \leqslant \alpha(t)+\beta(t) \mu+\gamma(t)|y(t)| \leqslant \alpha^{*}+\beta^{*} \mu+\gamma^{*}|y(t)|,
$$

where $\alpha^{*}=\sup _{t \in \mathcal{J}_{m}} \alpha(t), \beta^{*}=\sup _{t \in \mathcal{J}_{\mathfrak{m}}} \beta(t)$, and $\gamma^{*}=\sup _{t \in \mathcal{J}_{\mathfrak{m}}} \gamma(t)<1$. From above last result, we get

$$
|y(t)| \leqslant \frac{\alpha^{*}+\beta^{*} \mu}{1-\gamma^{*}}=M \text {. }
$$

And by $\left(\mathrm{H}_{7}\right)$, we have $|\sigma(\mathrm{t}, \mathrm{u})| \leqslant \mathfrak{I}$. Thus by $\left(\mathrm{H}_{5}\right)$, $\left(\mathrm{H}_{6}\right)$, and $\left(\mathrm{H}_{7}\right)$, (3.7) becomes

$$
|(\digamma \mathfrak{u})(\mathrm{t})| \leqslant \frac{M T^{p}}{\Gamma(p+1)}+\frac{q M T^{p}}{\Gamma(p+1)}+q\left(\mu N+N^{*}\right)+\frac{\mathfrak{I T}^{p}}{\Gamma(p+1)}=Q .
$$

Similarly for $t \in \mathcal{J}_{0}$, we have

$$
|(\digamma \mathrm{u})(\mathrm{t})| \leqslant \frac{M T^{p}}{\Gamma(p+1)}+\frac{\mathfrak{I}^{p}}{\Gamma(p+1)}=Q^{*} .
$$

Step 3. $\digamma$ maps bounded set into equi-continuous set of $\mathfrak{B}$.

Let $t_{1}, t_{2} \in \mathcal{J}_{m}$ with $t_{1}<t_{2}$ and let $B_{\mu}$ be a bounded set as in the second step. Then for $u \in B_{\mu}$, we have

$$
\begin{aligned}
\left|(\digamma \mathrm{u})\left(\mathrm{t}_{2}\right)-(\digamma \mathrm{u})\left(\mathrm{t}_{1}\right)\right| \leqslant & \int_{\mathrm{t}_{\mathrm{m}}}^{\mathrm{t}_{1}}\left|\frac{\left(\mathrm{t}_{2}-\zeta\right)^{p-1}-\left(\mathrm{t}_{1}-\zeta\right)^{p-1}}{\Gamma(p)} \mathrm{y}(\zeta)\right| \mathrm{d} \zeta+\int_{\mathrm{t}_{1}}^{\mathrm{t}_{2}}\left|\frac{\left(\mathrm{t}_{2}-\zeta\right)^{p-1}}{\Gamma(p)} \mathrm{y}(\zeta)\right| \mathrm{d} \zeta \\
& +\sum_{0<\mathrm{t}_{\mathrm{m}}<\mathrm{t}_{2}-\mathrm{t}_{1}}\left|\mathrm{I}_{\mathrm{m}}\left(\mathrm{u}\left(\mathrm{t}_{\mathrm{m}}\right)\right)\right| \\
& \leqslant \frac{M}{\Gamma(\mathrm{p}+1)}\left[\left(\mathrm{t}_{2}-\mathrm{t}_{\mathrm{m}}\right)^{p}-\left(\mathrm{t}_{2}-\mathrm{t}_{1}\right)^{p}-\left(\mathrm{t}_{1}-\mathrm{t}_{\mathrm{m}}\right)^{p}\right]+\left(\mathrm{t}_{2}-\mathrm{t}_{1}\right)\left(\mathrm{N}\|\mathrm{u}\|_{\mathrm{PC}}+\mathrm{N}^{*}\right) .
\end{aligned}
$$

Similarly if $t_{1}, t_{2} \in \mathcal{J}_{0}$, with $t_{1}<t_{2}$ and $u \in B_{\mu}$, we have

$$
\begin{aligned}
\left|(\digamma u)\left(t_{2}\right)-(\digamma u)\left(t_{1}\right)\right| & \leqslant\left|\int_{0}^{t_{1}}\left(\frac{\left(t_{2}-\zeta\right)^{p-1}}{\Gamma(p)}-\frac{\left(t_{1}-\zeta\right)^{p-1}}{\Gamma(p)}\right) y(\zeta) d \zeta\right|+\left|\int_{t_{1}}^{t_{2}} \frac{\left(t_{2}-\zeta\right)^{p-1}}{\Gamma(p)} y(\zeta) d \zeta\right| \\
& \leqslant \frac{M}{\Gamma(p+1)}\left[t_{2}^{p}-t_{1}^{p}+2\left(t_{2}-t_{1}\right)^{p}\right] .
\end{aligned}
$$

We see, the right-hand sides of (3.8) and (3.9) tend to zero as $t_{1} \rightarrow t_{2}$. Therefore, as a result of the above three steps and Arzelà-Ascoli theorem, we deduce that $\digamma: \mathfrak{B} \rightarrow \mathfrak{B}$ is completely continuous.

Step 4. A priori bound. Now in the final step, we show that the set defined by

$$
\mathrm{Z}=\{\mathfrak{u} \in \mathfrak{B}: \mathfrak{u}=\xi(\digamma \mathfrak{u}) \text { for some } 0<\xi<1\}
$$

is bounded. Let $u \in Z$, then for some $0<\xi<1, u=\xi(\digamma u)$. Therefore for $t \in \mathcal{J}$, we have

$$
\begin{aligned}
u(t)= & \xi \int_{t_{m}}^{t} \frac{(t-\zeta)^{p-1}}{\Gamma(p)} y(\zeta) d \zeta+\xi \sum_{0<t_{m}<t} \int_{t_{m-1}}^{t_{m}} \frac{\left(t_{m}-\zeta\right)^{p-1}}{\Gamma(p)} y(\zeta) d \zeta \\
& +\xi \sum_{0<t_{m}<t} I_{m}\left(u\left(t_{m}\right)\right)+\xi \int_{0}^{T} \frac{(T-\zeta)^{p-1}}{\Gamma(p)} \sigma(\zeta, u(\zeta)) d \zeta \\
\leqslant & \int_{t_{m}}^{t} \frac{(t-\zeta)^{p-1}}{\Gamma(p)} y(\zeta) d \zeta+\sum_{0<t_{\mathfrak{m}}<t} \int_{t_{m-1}}^{t_{m}} \frac{\left(t_{m}-\zeta\right)^{p-1}}{\Gamma(p)} y(\zeta) d \zeta \\
& +\sum_{0<t_{m}<t} I_{m}\left(u\left(t_{m}\right)\right)+\int_{0}^{T} \frac{(T-\zeta)^{p-1}}{\Gamma(p)} \sigma(\zeta, u(\zeta)) d \zeta .
\end{aligned}
$$


Also, we have $|y(t)| \leqslant \frac{\alpha^{*}+\beta^{*}\|\mathfrak{u}\|_{P C}}{1-\gamma^{*}}=M$. Thus (3.10) becomes

$$
\begin{aligned}
|\mathfrak{u}(\mathrm{t})| \leqslant & \frac{\left(\alpha^{*}+\beta^{*}\|u\|_{P C}\right)}{\left(1-\gamma^{*}\right) \Gamma(p)} \int_{t_{m}}^{t}(t-\zeta)^{p-1} d \zeta+\frac{\left(\alpha^{*}+\beta^{*}\|\mathfrak{u}\|_{P C}\right)}{\left(1-\gamma^{*}\right) \Gamma(p)} \sum_{0<t_{m}<t} \int_{t_{m-1}}^{t_{m}}\left(t_{m}-\zeta\right)^{p-1} d \zeta \\
& +q\left(N\|u\|_{P C}+N^{*}\right)+\frac{\mathfrak{I}}{\Gamma(p)} \int_{0}^{T}(T-\zeta)^{p-1} d \zeta, \\
\|u\|_{P C} \leqslant & \frac{\frac{\left((q+1) \alpha^{*} T^{p}\right.}{\left(1-\gamma^{*}\right) \Gamma(p+1)}+q N^{*}+\frac{\mathfrak{s} T^{p}}{\Gamma(p+1)}}{1-q N-\frac{\beta^{*} T^{p}(q+1)}{\left(1-\gamma^{*}\right) \Gamma(p+1)}}=\mathbf{M} .
\end{aligned}
$$

It means that the set $Z$ is bounded. Thus via the Schaefer's fixed point theorem, we conclude that $\digamma$ has a fixed point which is the corresponding solution of the proposed problem (1.1).

\section{Ulam-Hyers stability analysis}

In this section, we obtain some sufficient conditions for the problem (1.1) to satisfy the definitions of various types of Ulam-Hyers stabilty.

Theorem 4.1. If the assumptions $\left(\mathrm{H}_{1}\right)-\left(\mathrm{H}_{3}\right)$ and the inequality (3.4) are satisfied, then the problem (1.1) is UlamHyers stable and consequently generalized Ulam-Hyers stable.

Proof. Let $w \in \mathfrak{B}$ be a solution of the inequality (2.1) and let $u$ be the unique solution of the following problem

$$
\left\{\begin{array}{l}
C_{\mathcal{D}^{p}} u(t)=g\left(t, u(t), C{ }^{p} \mathfrak{p}(t)\right), t \neq t_{m} \in \mathcal{J}=[0, T], \text { for } m=1,2, \ldots, q \\
u(0)=\int_{0}^{T} \frac{(T-\zeta)^{p-1}}{\Gamma(p)} \sigma(\zeta, u(\zeta)) d \zeta \\
\triangle u\left(t_{m}\right)=I_{m}\left(u\left(t_{m}\right), m=1,2, \ldots, q\right.
\end{array}\right.
$$

By Lemma 3.1, we have for each $t \in \mathcal{J}_{m}$

$$
\begin{aligned}
u(t)= & \int_{t_{m}}^{t} \frac{(t-\zeta)^{p-1}}{\Gamma(p)} y(\zeta) d \zeta+\sum_{i=1}^{m} \int_{t_{i-1}}^{t_{i}} \frac{\left(t_{i}-\zeta\right)^{p-1}}{\Gamma(p)} y(\zeta) d \zeta \\
& +\sum_{i=1}^{m} I_{i}\left(u\left(t_{i}\right)\right)+\int_{0}^{T} \frac{(T-\zeta)^{p-1}}{\Gamma(p)} \sigma(\zeta, u(\zeta)) d \zeta, t \in \mathcal{J}_{m}, m=1,2, \ldots, q,
\end{aligned}
$$

where $y \in C(\mathcal{J}, \mathbb{R})$ and is given by $y(t)=g(t, u(t), y(t))$. Since $w$ is a solution of inequality (2.1), hence by Remark 2.12, we get

$$
\left\{\begin{array}{l}
{ }^{C} \mathcal{D}^{p} w(t)=g\left(t, w(t),{ }^{C} \mathcal{D}^{p} w(t)\right)+\varpi(t), t \in J_{m}, m=1,2, \ldots, q \\
w(0)=\int_{0}^{T} \frac{(T-\zeta)^{p-1}}{\Gamma(p)} \sigma(\zeta, w(\zeta)) d \zeta \\
\triangle w\left(t_{m}\right)=I_{m}\left(w\left(t_{m}\right)\right)+\varpi_{m}, m=1,2, \ldots, q .
\end{array}\right.
$$

Obviously the solution of (4.1) will be

$$
w(t)=\left\{\begin{array}{l}
\frac{1}{\Gamma(p)} \int_{0}^{t}(t-\zeta)^{p-1} x(\zeta) d \zeta+\frac{1}{\Gamma(p)} \int_{0}^{t}(t-\zeta)^{p-1} \varpi(\zeta) d \zeta+\int_{0}^{T} \frac{(T-\zeta)^{p-1}}{\Gamma(p)} \sigma(\zeta, w(\zeta)) d \zeta, t \in J_{0} \\
\int_{t_{m}}^{t} \frac{(t-\zeta)^{p-1}}{\Gamma(p)} x(\zeta) d \zeta+\int_{t_{m}}^{t} \frac{(t-\zeta)^{p-1}}{\Gamma(p)} \varpi(\zeta) d \zeta+\sum_{i=1}^{m} \int_{t_{i-1}}^{t_{i}} \frac{\left(t_{i}-\zeta\right)^{p-1}}{\Gamma(p)} x(\zeta) d \zeta \\
\quad+\sum_{i=1}^{m} \int_{t_{i-1}}^{t_{i}} \frac{\left(t_{i}-\zeta\right)^{p-1}}{\Gamma(p)} \varpi(\zeta) d \zeta+\sum_{i=1}^{m} I_{i}\left(w\left(t_{i}\right)\right)+\sum_{i=1}^{m} \varpi_{i} \\
\quad+\int_{0}^{T} \frac{(T-\zeta)^{p-1}}{\Gamma(p)} \sigma(\zeta, w(\zeta)) d \zeta, t \in J_{m}, m=1,2, \ldots, q
\end{array}\right.
$$


where $x \in C(\mathcal{J}, \mathbb{R})$ and is given by

$$
x(t)=g(t, w(t), x(t)) .
$$

Therefore, for each $t \in \mathcal{J}_{m}$, we have the following

$$
\begin{aligned}
|w(t)-u(t)| \leqslant & \int_{t_{m}}^{t} \frac{(t-\zeta)^{p-1}}{\Gamma(p)}|x(\zeta)-y(\zeta)| d \zeta+\int_{t_{m}}^{t} \frac{(t-\zeta)^{p-1}}{\Gamma(p)}|\varpi(\zeta)| d \zeta \\
& +\sum_{i=1}^{m} \int_{t_{i-1}}^{t_{i}} \frac{\left(t_{i}-\zeta\right)^{p-1}}{\Gamma(p)}|x(\zeta)-y(\zeta)| d \zeta+\sum_{i=1}^{m} \int_{t_{i-1}}^{t_{i}} \frac{\left(t_{i}-\zeta\right)^{p-1}}{\Gamma(p)}|\varpi(\zeta)| d \zeta \\
& +\sum_{i=1}^{m}\left|I_{i}\left(w\left(t_{i}\right)\right)-I_{i}\left(u\left(t_{i}\right)\right)\right|+\sum_{i=1}^{m}\left|\varpi_{i}\right| \\
& +\int_{0}^{T} \frac{(T-\zeta)^{p-1}}{\Gamma(p)}|\sigma(\zeta, w(\zeta))-\sigma(\zeta, u(\zeta))| d \zeta, t \in J_{m}, m=1,2, \ldots, q .
\end{aligned}
$$

By $\left(\mathrm{H}_{2}\right)$ we get

$$
\|x-y\| \leqslant \frac{K_{g}}{1-L_{g}}\|w-u\|_{P C} .
$$

Hence by $\left(\mathrm{H}_{2}\right),\left(\mathrm{H}_{3}\right),\left(\mathrm{H}_{4}\right)$, and (i) of Remark 2.12, we get

$$
\begin{aligned}
\|w-u\|_{P C} \leqslant & \frac{K_{g}\|w-u\|_{P C}}{1-L_{g}} \int_{t_{m}}^{t} \frac{(t-\zeta)^{p-1}}{\Gamma(p)} d \zeta+\epsilon \int_{t_{m}}^{t} \frac{(t-\zeta)^{p-1}}{\Gamma(p)} d \zeta \\
& +\frac{K_{g}\|w-u\|_{P C}}{1-L_{g}} \sum_{i=1}^{m} \int_{t_{i-1}}^{t_{i}} \frac{\left(t_{i}-\zeta\right)^{p-1}}{\Gamma(p)} d \zeta \\
& +\epsilon \sum_{i=1}^{m} \int_{t_{i-1}}^{t_{i}} \frac{\left(t_{i}-\zeta\right)^{p-1}}{\Gamma(p)} d \zeta+\|w-u\|_{P C} \sum_{i=1}^{m} L_{I}+\sum_{i=1}^{m} \epsilon \\
& +L_{\sigma}\|w-u\|_{P C} \int_{0}^{T} \frac{(T-\zeta)^{p-1}}{\Gamma(p)} d \zeta, t \in \mathcal{J}_{m}, m=1,2, \ldots, q \\
\leqslant & \epsilon\left(\frac{T^{p}}{\Gamma(p+1)}(1+q)+q\right) \\
& +\left[\frac{K_{g} T^{p}}{\left(1-L_{g}\right) \Gamma(p+1)}+\frac{L_{\sigma} T^{p}}{\Gamma(p+1)}+q\left(\frac{K_{g} T^{p}}{\left(1-L_{g}\right) \Gamma(p+1)}+L_{I}\right)\right]\|w-u\|_{P C},
\end{aligned}
$$

which implies that

$$
\|w-u\|_{P C} \leqslant \frac{\epsilon\left(\frac{T^{p}}{\Gamma(p+1)}(1+q)+q\right)}{1-\left[\frac{K_{g} T^{p}}{\left(1-L_{g}\right) \Gamma(p+1)}+\frac{L_{\sigma} T^{p}}{\Gamma(p+1)}+q\left(\frac{K_{g} T^{p}}{\left(1-L_{g}\right) \Gamma(p+1)}+L_{I}\right)\right]} .
$$

Similarly for $t \in \mathcal{J}_{0}$, we obtain

$$
\|w-u\|_{P C} \leqslant \frac{\epsilon \frac{T^{p}}{\Gamma(p+1)}}{1-\frac{T^{p}}{\Gamma(p+1)}\left(\frac{K_{g}}{1-L_{g}}+L_{\sigma}\right)}
$$

Combining (4.2) and (4.3), for $t \in \mathcal{J}$, we have

$$
\|w-u\|_{P C} \leqslant \epsilon\left[\frac{\left(\frac{T^{p}}{\Gamma(p+1)}(1+q)+q\right)}{1-\left[\frac{K_{g} T^{p}}{\left(1-L_{g}\right) \Gamma(p+1)}+\frac{L_{\sigma} T^{p}}{\Gamma(p+1)}+q\left(\frac{K_{g} T^{p}}{\left(1-L_{g}\right) \Gamma(p+1)}+L_{I}\right)\right]}+\frac{\frac{T^{p}}{\Gamma(p+1)}}{1-\frac{T^{p}}{\Gamma(p+1)}\left(\frac{L_{g}}{1-L_{g}}+L_{\sigma}\right)}\right] .
$$


Thus

$$
\|w-u\|_{P C} \leqslant c_{g, p, q, \sigma} \epsilon
$$

where

$$
c_{g, p, q, \sigma}=\left[\frac{\left(\frac{T^{p}}{\Gamma(p+1)}(1+q)+q\right)}{1-\left[\frac{K_{g} T^{p}}{\left(1-L_{g}\right) \Gamma(p+1)}+\frac{L_{\sigma} T^{p}}{\Gamma(p+1)}+q\left(\frac{K_{g} T^{p}}{\left(1-L_{g}\right) \Gamma(p+1)}+L_{I}\right)\right]}+\frac{\frac{T^{p}}{\Gamma(p+1)}}{1-\frac{T^{p}}{\Gamma(p+1)}\left(\frac{K_{g}}{1-L_{g}}+L_{\sigma}\right)}\right] .
$$

Hence the problem (1.1) is Ulam-Hyers stable. Moreover if we set $\theta(\epsilon)=c_{g, p, q, \sigma}(\epsilon) ; \theta(0)=0$, then the problem (1.1) is generalized Ulam-Hyers stable.

Now for the next result we assume that

$\left(\mathrm{H}_{8}\right)$ there exists a nondecreasing function $\varphi \in \mathrm{PC}\left(\mathcal{J}, \mathbb{R}_{+}\right)$and there exists $\lambda_{\varphi}>0$ such that

$$
\mathrm{I}^{\mathrm{p}} \varphi(\mathrm{t}) \leqslant \lambda_{\varphi} \varphi(\mathrm{t}) \text { for each } \mathrm{t} \in \mathcal{J} \text {. }
$$

Theorem 4.2. Assume that $\left(\mathrm{H}_{1}\right)-\left(\mathrm{H}_{4}\right),\left(\mathrm{H}_{8}\right)$, and inequality (3.4) are satisfied, then the problem (1.1) is UlamHyers-Rassias stable with respect to $(\psi, \varphi)$, consequently generalized Ulam-Hyers-Rassias stable.

Proof. Let $w \in \mathcal{J}_{\mathrm{m}}$ be a solution of the inequality (2.3) and let $u$ be a unique solution of the following problem

$$
\left\{\begin{array}{l}
{ }^{C} \mathcal{D}^{p} u(t)=g\left(t, u(t),{ }^{C} \mathcal{D}^{p} u(t)\right), t \neq t_{m} \in \mathcal{J}=[0, T], \text { for } m=1,2, \ldots, q, \\
u(0)=\int_{0}^{T} \frac{(T-\zeta)^{p-1}}{\Gamma(p)} \sigma(\zeta, u(\zeta)) d \zeta \\
\triangle u\left(t_{m}\right)=I_{m}\left(u\left(t_{m}\right), m=1,2, \ldots, q .\right.
\end{array}\right.
$$

From the proof of Theorem 4.1, for each $t \in \mathcal{J}_{m}$, we obtain

$$
\begin{aligned}
|w(t)-u(t)| \leqslant & \int_{t_{m}}^{t} \frac{(t-\zeta)^{p-1}}{\Gamma(p)}|x(\zeta)-y(\zeta)| d \zeta+\int_{t_{m}}^{t} \frac{(t-\zeta)^{p-1}}{\Gamma(p)}|\varpi(\zeta)| d \zeta \\
& +\sum_{i=1}^{m} \int_{t_{i-1}}^{t_{i}} \frac{\left(t_{i}-\zeta\right)^{p-1}}{\Gamma(p)}|x(\zeta)-y(\zeta)| d \zeta+\sum_{i=1}^{m} \int_{t_{i-1}}^{t_{i}} \frac{\left(t_{i}-\zeta\right)^{p-1}}{\Gamma(p)}|\varpi(\zeta)| d \zeta \\
& +\sum_{i=1}^{m}\left|I_{i}\left(w\left(t_{i}\right)\right)-I_{i}\left(u\left(t_{i}\right)\right)\right|+\sum_{i=1}^{m}\left|\varpi_{i}(t)\right| \\
& +\int_{0}^{T} \frac{(T-\zeta)^{p-1}}{\Gamma(p)}|\sigma(\zeta, w(\zeta))-\sigma(\zeta, u(\zeta))| d \zeta, t \in J_{m}, m=1,2, \ldots, q
\end{aligned}
$$

By $\left(\mathrm{H}_{2}\right)$ we get

$$
|x(t)-y(t)| \leqslant \frac{K_{g}}{1-L_{g}}\|w-u\|_{P C} .
$$

Hence by $\left(\mathrm{H}_{2}\right),\left(\mathrm{H}_{3}\right),\left(\mathrm{H}_{4}\right)$, and (i) of Remark 2.14, we get

$$
\begin{aligned}
|w(t)-u(t)| \leqslant & \frac{K_{g}\|w-u\|_{P C}}{1-L_{g}} \int_{t_{m}}^{t} \frac{(t-\zeta)^{p-1}}{\Gamma(p)} d \zeta+\epsilon \int_{t_{m}}^{t} \frac{(t-\zeta)^{p-1}}{\Gamma(p)} \varphi(\zeta) d \zeta \\
& +\frac{K_{g}\|w-u\|_{P C}}{1-L_{g}} \sum_{i=1}^{m} \int_{t_{i-1}}^{t_{i}} \frac{\left(t_{i}-\zeta\right)^{p-1}}{\Gamma(p)} d \zeta+\epsilon \sum_{i=1}^{m} \int_{t_{i-1}}^{t_{i}} \frac{\left(t_{i}-\zeta\right)^{p-1}}{\Gamma(p)} \varphi(\zeta) d \zeta \\
& +\|w-u\|_{P C} \sum_{i=1}^{m} L_{I}+\epsilon \sum_{i=1}^{m} \psi+L_{\sigma}\|w-u\|_{P C} \int_{0}^{T} \frac{(T-\zeta)^{p-1}}{\Gamma(p)} d \zeta, t \in \mathcal{J}_{m}, m=1,2, \ldots, q
\end{aligned}
$$


Using $\left(\mathrm{H}_{8}\right)$, we get

$$
\begin{aligned}
|w(t)-u(t)| \leqslant & \in\left(\lambda_{\varphi} \varphi(t)(1+q)+q \psi\right) \\
& +\left[\frac{K_{g} T^{p}}{\left(1-L_{g}\right) \Gamma(p+1)}+\frac{L_{\sigma} T^{p}}{\Gamma(p+1)}+q\left(\frac{K_{g} T^{p}}{\left(1-L_{g}\right) \Gamma(p+1)}+L_{I}\right)\right]\|w-u\|_{P C} \\
\leqslant & \epsilon(\varphi(t)+\psi)\left(\lambda_{\varphi}(1+q)+q\right) \\
& +\left[\frac{K_{g} T^{p}}{\left(1-L_{g}\right) \Gamma(p+1)}+\frac{L_{\sigma} T^{p}}{\Gamma(p+1)}+q\left(\frac{K_{g} T^{p}}{\left(1-L_{g}\right) \Gamma(p+1)}+L_{I}\right)\right]\|w-u\|_{P C} .
\end{aligned}
$$

From which we have

$$
\|w-u\|_{P C} \leqslant \frac{\epsilon(\varphi(t)+\psi)\left(\lambda_{\varphi}(1+q)+q\right)}{1-\left[\frac{K_{g} T^{p}}{\left(1-L_{g}\right) \Gamma(p+1)}+\frac{L_{\sigma} T^{p}}{\Gamma(p+1)}+q\left(\frac{K_{g} T^{p}}{\left(1-L_{g}\right) \Gamma(p+1)}+L_{I}\right)\right]} .
$$

Similarly for $t \in \mathcal{J}_{0}$, we obtain

$$
\|w-u\|_{P C} \leqslant \frac{\epsilon \lambda_{\varphi} \varphi(t)}{1-\frac{T^{p}}{\Gamma(p+1)}\left(\frac{K_{g}}{1-L_{g}}+L_{\sigma}\right)} .
$$

Combining both results of (4.4) and (4.5), we have for $t \in \mathcal{J}$

$$
\begin{aligned}
\|w-u\|_{P C} \leqslant & \in(\varphi(t)+\psi) \\
& \times\left[\frac{\left(\lambda_{\varphi}(1+q)+q\right)}{1-\left[\frac{K_{g} T^{p}}{\left(1-L_{g}\right) \Gamma(p+1)}+\frac{L_{\sigma} T^{p}}{\Gamma(p+1)}+q\left(\frac{K_{g} T^{p}}{\left(1-L_{g}\right) \Gamma(p+1)}+L_{I}\right)\right]}+\frac{\lambda_{\varphi}}{1-\frac{T^{p}}{\Gamma(p+1)}\left(\frac{K_{g}}{1-L_{g}}+L_{\sigma}\right)}\right] .
\end{aligned}
$$

Thus

$$
\|w-u\|_{P C} \leqslant c_{g, p, q, \sigma, \varphi} \in(\varphi(t)+\psi),
$$

where

$$
c_{g, p, q, \sigma, \varphi}=\left[\frac{\left(\lambda_{\varphi}(1+q)+q\right)}{1-\left[\frac{\mathrm{K}_{g} T^{p}}{\left(1-L_{g}\right) \Gamma(p+1)}+\frac{L_{\sigma} T^{p}}{\Gamma(p+1)}+q\left(\frac{\mathrm{K}_{\mathrm{g}} \mathrm{T}^{p}}{\left(1-\mathrm{L}_{\mathrm{g}}\right) \Gamma(\mathrm{p}+1)}+\mathrm{L}_{\mathrm{I}}\right)\right]}+\frac{\lambda_{\varphi}}{1-\frac{\mathrm{Tp}^{p}}{\Gamma(\mathrm{p}+1)}\left(\frac{\mathrm{K}_{\mathrm{g}}}{1-\mathrm{L}_{\mathrm{g}}}+\mathrm{L}_{\sigma}\right)}\right] .
$$

Thus the problem (1.1) is Ulam-Hyers-Rassias stable. Hence it is also obvious that the proposed problem (3.1) is generalized Ulam-Hyers-Rassias stable.

\section{Example}

To justify our established results, we provide the following example.

\section{Example 5.1.}

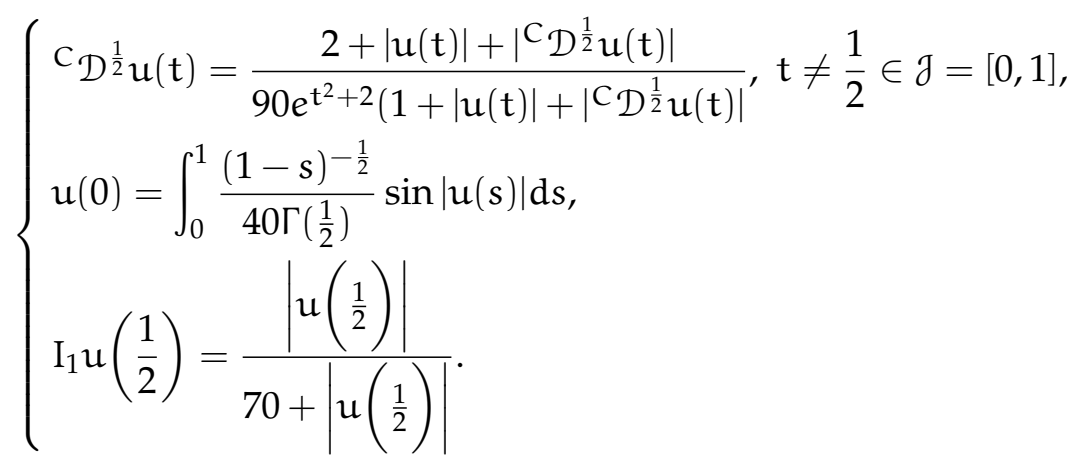


Here $p=\frac{1}{2}, \mathfrak{J}_{0}=\left[0, \frac{1}{2}\right], \mathfrak{J}_{1}=\left(\frac{1}{2}, 1\right], \mathrm{t}_{0}=0, \mathrm{t}_{1}=\frac{1}{2}, \mathrm{q}=1$. Also

$$
g(t, u, w)=\frac{2+|u(t)|+\left|{ }^{C} \mathcal{D}^{\frac{1}{2}} u(t)\right|}{90 e^{t^{2}+2}\left(1+|u(t)|+\left|{ }^{C} \mathcal{D}^{\frac{1}{2}} u(t)\right|\right.}, t \in[0,1], u, w \in \mathbb{R}, \sigma(t, u(t))=\frac{1}{40} \sin |u(t)|
$$

is continuous. Therefore, for $u, w, \bar{u}, \bar{w} \in \mathbb{R}$, we have

$$
|g(t, u, w)-g(t, \bar{u}, \bar{w})| \leqslant \frac{1}{90 e^{2}}[|u-\bar{u}|+|w-\bar{w}|] .
$$

So we have $\mathrm{K}_{\mathrm{g}}=\mathrm{L}_{\mathrm{g}}=\frac{1}{90 \mathrm{e}^{2}}$. Thus $\left(\mathrm{H}_{2}\right)$ holds and also we see

from which we have

$$
g(t, u, w) \leqslant \frac{1}{90 e^{2}}\left(2+|u(t)|+\left|{ }^{C} \mathcal{D}^{\frac{1}{2}} u(t)\right|\right) \text { for every } t \in \mathcal{J},
$$

$$
\alpha(t)=\frac{1}{45 e^{t^{2}+2}}, \beta(t)=\gamma(t)=\frac{1}{90 e^{t^{2}+2}}
$$

which implies that $\alpha^{*}=\frac{1}{45 e^{2}}, \beta^{*}=\frac{1}{90 e^{2}}, \gamma^{*}=\frac{1}{90 e^{2}}$. Further, we see that

$$
\left|\mathrm{I}_{1} \mathrm{u}\left(\frac{1}{2}\right)\right| \leqslant \frac{1}{70}\left|\mathrm{u}\left(\frac{1}{2}\right)\right|+1
$$

from which, one can see that $\mathrm{N}=\frac{1}{70}, \mathrm{~N}^{*}=1$ and also

$$
\left|\mathrm{I}_{1} \mathrm{u}\left(\frac{1}{2}\right)-\mathrm{I}_{1} \overline{\mathrm{u}}\left(\frac{1}{2}\right)\right| \leqslant \frac{1}{70}|\mathrm{u}-\overline{\mathrm{u}}| .
$$

Obviously

$$
q N+\frac{\beta^{*} T^{p}(q+1)}{\left(1-\gamma^{*}\right) \Gamma(p+1)}=\frac{1}{70}+\frac{\frac{2}{90 e^{2}}}{\left(1-\frac{1}{90 e^{2}}\right) \Gamma\left(\frac{3}{2}\right)}<1 .
$$

Thus, thanks to Theorem 3.3, the given problem (5.1) has at least one solution. Further

$$
\frac{\mathrm{K}_{\mathrm{g}} \mathrm{T}^{\mathrm{p}}}{\left(1-\mathrm{L}_{\mathrm{g}}\right) \Gamma(\mathrm{p}+1)}+\frac{\mathrm{L}_{\sigma} \mathrm{T}^{\mathrm{p}}}{\Gamma(\mathrm{p}+1)}+\mathrm{q}\left(\frac{\mathrm{K}_{\mathrm{g}} \mathrm{T}^{\mathrm{p}}}{\left(1-\mathrm{L}_{\mathrm{g}}\right) \Gamma(\mathrm{p}+1)}+\mathrm{L}_{\mathrm{I}}\right)=\frac{4}{\left(90 e^{2}-1\right) \sqrt{\pi}}+\frac{1}{20 \sqrt{\pi}}+\frac{1}{70}=0.045695<1 .
$$

Thus by Theorem 3.2, the given problem (5.1) has a unique solution. Further the conditions of Theorem 4.1 are satisfied so the solution of the given problem (5.1) is Ulam-Hyers stable and generalized UlamHyers stable. Further it is also easy to check the conditions of Theorem 4.2 hold and thus the problem (5.1) is Ulam-Hyers-Rassias stable and consequently generalized Ulam-Hyers-Rassias stable.

\section{Conclusion}

With the help of nonlinear functional analysis and fixed point theorems of Banach and Schaefer, we have successfully developed some adequate conditions for the uniqueness and existence of solution to a nonlinear implicit type impulsive boundary value problem of FDEs. The obtained conditions ensure the existence of at least one solution to the proposed problem. Further different kinds of Ulam type stability have been investigated which is a new direction of analysis of nonlinear FDEs. The concerned stability is very much important from optimization and numerical point of view.

\section{Acknowledgment}

We are really thankful to the anonymous referees for their useful comments which improved the quality of this paper.

This project was financially supported by the Department of Mathematics, Faculty of Science, Universiti Putra Malaysia, 43400 UPM Serdang, Selangor, Malaysia and Institute For Mathematical Research, Universiti Putra Malaysia, 43400 UPM Serdang, Selangor, Malaysia. 


\section{References}

[1] H. Akça, R. Alassar, V. Covachev, Z. Covacheva, Continuous-time additive Hopfield-type neural networks with impulses, J. Math. Anal. Appl., 290 (2004), 436-451. 1

[2] A. Ali, B. Samet, K. Shah, R. A. Khan, Existence and stability of solution ta toppled systems of differential equations of non-integer order, Boundary Value Problems., 2017 (2017), 13 pages. 1

[3] Z. Ali, A. Zada, K. Shah, Existence and stability analysis of three point boundary value problem, Int. J. Appl. Comput. Math., 2017 (2017), 14 pages. 1

[4] S. András, J. J. Kolumbán, On the Ulam-Hyers stability of first order differential systems with nonlocal initial conditions, Nonlinear Anal., 82 (2013), 1-11. 1

[5] S. András, A. R. Mészáros, Ulam-Hyers stability of dynamic equations on time scales via Picard operators, Appl. Math. Comput., 219 (2013), 4853-4864. 1

[6] D. D. Bainov, M. B. Dimitrova, A. B. Dishliev, Oscillation of the bounded solutions of impulsive differential-difference equations of second order, Appl. Math. Comput., 114 (2000), 61-68. 1

[7] T. Bashiri, S. M. Vaezpour, C. Park, A coupled fixed point theorem and application to fractional hybrid differential problems, Fixed Point Theory and Appl., 2016 (2016), 11 pages. 1

[8] M. Benchohra, S. Bouriah, Existence and stability results for nonlinear boundary value problem for implicit differential equations of fractional order, Moroccan J. Pure Appl. Anal., 1 (2015), 22-37. 1

[9] M. Benchohra, J. Henderson, S. K. Ntouyas, Impulsive Differential Equations and Inclusions, Hindawi Publishing Corporation, New York, (2006). 1

[10] N. Brillouët-Belluot, J. Brzdęk, K. Ciepliński, On some recent developments in Ulam's type stability, Abstr. Appl. Anal., 2012 (2012), 41 pages. 1

[11] J. Brzdęk, S.-M. Jung, A note on stability of an operator linear equation of the second order, Abstr. Appl. Anal., 2011 (2011), 15 pages.

[12] M. Burger, N. Ozawa, A. Thom, On Ulam stability, Israel J. Math., 193 (2013), 109-129. 1

[13] L. O. Chua, L. Yang, Cellular Neural Network: Applications, IEEE Trans. circuits and systems, 35 (1988), 1273-1290. 1

[14] K. Ciepliński, Applications of fixed point theorems to the Hyers-Ulam stability of functional equations a survey, Ann. Funct. Anal., 3 (2012), 151-164. 1

[15] D. S. Cimpean, D. Popa, HyersUlam stability of Eulers equation, Appl. Math. Lett., 24 (2011), 1539-1543. 1

[16] A. Granas, J. Dugundji, Fixed Point Theory, Springer-Verlag, New York, (2003). 2.15, 2.16

[17] B. Hegyi, S.-M. Jung, On the stability of Laplaces equation, Appl. Math. Lett., 26 (2013), 549-552. 1

[18] S.-M. Jung, T. M. Rassias, Generalized Hyers-Ulam stability of Riccati differential equation, Math. Inequal. Appl., 11 (2008), 777-782. 1

[19] A. A. Kilbas, H. M. Srivastava, J. J. Trujillo, Theory and Applications of Fractional Differential Equations, Elsevier Science, Amsterdam, (2006). 2

[20] P. Kumama, A. Ali, K. Shah, R. A. Khan, Existence results and Hyers-Ulam stability to a class of nonlinear arbitrary order differential equations, J. Nonlinear Sci. Appl., 10 (2017), 2986-2997. 2, 2.17

[21] T.-X. Li, A. Zada, S. Faisal, Hyers-Ulam stability of nth order linear differential equations, J. Nonlinear Sci. Appl., 9 (2016), 2070-2075. 1

[22] H. V. Long, J. J. Nieto, N. T. K. Son, New approach to study nonlocal problems for differential systems and partial differential equations in generalized fuzzy metric spaces, Fuzzy Sets and Systems, 2016 (2016), 31 pages. 1

[23] H. V. Long, N. T. K. Son, N. V. Hoa, Fuzzy fractional partial differential equations in partially ordered metric spaces, Iran. J. Fuzzy Syst., 14 (2017), 107-126. 1

[24] H. V. Long, N. T. K. Son, H. T. T. Tam, The solvability of fuzzy fractional partial differential equations under caputo gh-differentiability, Fuzzy Sets and Systems, 309 (2017), 35-63. 1

[25] H. V. Long, N. T. K. Son, H. T. T. Tam, J. C. Yao, Ulam Stability for Fractional Partial Integro-Differential Equation with Uncertainty, Acta Mathematica Vietnamica, 2017 (2017), 26 pages. 1

[26] D. Popa, I. Raşa, On the HyersUlam stability of the linear differential equation, J. Math. Anal. Appl., 381 (2011), 530-537. 1

[27] I. A. Rus, Ulam stability of ordinary differential equations, Stud. Univ. Babeş. Bolyai Math., 54 (2009), 125-133. 1

[28] M. Shoaib, M. Sarwara, K. Shaha, P. Kumam, Fixed point results and its applications to the systems of non-linear integral and differential equations of arbitrary order, J. Nonlinear Sci. Appl., 9 (2016), 4949-4962. 1

[29] V. E. Tarasov Fractional Dynamics: Application of Fractional calculus to Dynamics of Particles, Fields and Media, Springer, New York, (2010). 1

[30] A. Vinodkumar, K. Malar, M. Gowrisankar, P. Mohankumar, Existence, uniqueness and stability of random impulsive fractional differential equations, Acta Math. Sci., 36 (2016), 428-442. 1

[31] J. Wang, M. Fečikan, Y. Zhou, Ulams type stability of impulsive ordinary differential equations, J. Math. Anal. Appl., 395 (2012), 258-264. 1

[32] J.-R. Wang, Y. Zhang, A class of nonlinear differential equations with fractional integrable impulses, Commun. Nonlinear Sci. Numer Simul., 19 (2014), 3001-3010. 1 
[33] J.-R. Wang, Y. Zhang, Existence and stability of solutions to nonlinear impulsive differential equations in -normedspaces, Electron. J. Differential Equations, 2014 (2014), 10 pages. 1, 2.6, 2.7, 2.9, 2.10

[34] J.-R. Wang, Y. Zhou, W. Wei, Study in fractional differential equations by means of topological degree methods, Numer. Funct. Anal. Optim., 33 (2012), 216-238. 1, 2

[35] T. Z. Xu, On the stability of multi-Jensen mappings in $\beta$-normed spaces, Appl. Math. Lett., 25 (2012), 1866-1870. 1

[36] X.-J. Yang, C.-D. Li, T.-W. Huang, Q.-K. Song, Mittag-Leffler stability analysis of nonlinear fractional-order systems with impulses, Appl. Math. Comput., 293 (2017), 416-422. 1

[37] A. Zada, S. Faisal, Y.-J. Li, On the Hyers-Ulam stability of first-order impulsive delay differential equations, J. Funct. Spaces, 2016 (2016), 6 pages. 1

[38] A. Zada, O. Shah, R. Shah, Hyers-Ulam stability of nonautonomous systems in terms of bounded-ness of Cauchy problems, Appl. Math. Comput., 271 (2015), 512-518. 1

[39] S. Zavalishchin, A. Sesekin, Impulsive processes: models and applications, Nauka, Moskow, (1991). 1

[40] S. Zhang, Positive solutions for boundary value problem of nonlinear fractional differential equations, Electron. J. Differential Equations, 2006 (2006), 12 pages. 2 